\title{
NEWS IN PATHOGENESIS OF CHRONIC VENOUS INSUFFICIENCY
}

\author{
Mazuchova $\mathrm{J}^{1}$, Pec $\mathbf{M}^{1}$, Halasova $\mathrm{E}^{1}$, Valentova $\mathrm{V}^{1}$, Sarlinova $\mathbf{M}^{1}$, Mazuch $\mathrm{J}^{2}$, Zelnik $\mathrm{S}^{3}$ \\ Comenius University in Bratislava, Jessenius Faculty of Medicine in Martin and University Hospital in Martin \\ ${ }^{1}$ Department of Medical Biology, ${ }^{2}$ Clinic of Surgery and Transplant Center, ${ }^{3}$ ZILPO, s.r.o., Žilina
}

\begin{abstract}
This review article is concentrating on the news in the pathophysiology of chronic venous insufficiency (CVD). Despite ongoing progress in understanding the molecular aspects of CVD the exact mechanism of its development remains unclear. Many different factors may play role in the pathogenesis of CVD, including changes in hydrostatic pressure, valvular incompetence, increased capillary permeability, endothelial dysfunction, activation of leukocytes, deep venous obstruction, capillary microthrombosis, ineffective function of calf muscle pump, biochemical and structural changes in the vessel wall, extracellular matrix alteration, and several other mechanisms. A better understanding of the pathophysiology is an important step in the finding of new potential treatment.
\end{abstract}

Key words: chronic venous insufficiency, varicose veins, skin changes, leg ulcers, pathophysiology

\section{INTRODUCTION}

Chronic venous disease (CVD) affects approximately one-third of the adult Slovak population (1). It is one of the most common disorders of the venous system of the lower extremities where persistent ambulatory venous hypertension leads to various pathologies. If it is left untreated it can lead to pain, varicose veins development, edema, hyperpigmentation, lipodermatosclerosis, venous eczema, atrophie blanche, healed or active leg ulcers and venous thrombosis $(2,3,4)$. A high portion of uncomplicated varicose veins (C2) can progress to higher clinical stages (C3-C6). Rabe and Pannier (2015) in their long-term spotted that the progression rate of varicose veins reaches approximately $4.3 \%$ of all CVD patients per year $(5,6)$.

The exact mechanism of CVD development remains unclear. Current experimental studies attempt to create an unifying theory about the pathophysiology of venous reflux and the manifestation of venous hypertension and CVD $(7,8,9)$. Early theories assumed that varicose veins arose as aftereffects of valvular incompetence and venous hypertension. Unfortunately, there is little evidence of a constitutive valvular abnormality in primary venous disease. In addition, these theories cannot explain why are truncal varicosities often found below properly functioning valves, why normal valves are often seen between those exhibiting varices, or why dilation often precedes valvular incompetence (10). That's why in the past decade, there has been a shift from initial theories based purely on mechanical factors to the hypotheses pointing to complex molecular changes leading to the histologic changes in the vessel wall and extracellular matrix composition (11).

Many different factors may play role in the pathogenesis of CVD, including changes in hydrostatic pressure, valvular incompetence, increased capillary permeability, endothelial dysfunction, activation of leukocytes, deep venous obstruction, capillary microthrombosis, ineffective function of calf muscle pump, biochemical and structural changes in the vessel wall, extracellular matrix alteration, and several other mechanisms $(12,13)$.

The veins of the lower extremities are organized into three systems - superficial, deep, and perforator venous system. Perforating veins connect the superficial veins to the deep veins

Ad dress for corres pond e n c e:

Mgr. Jana Mazuchová, Mala Hora 4, 036 01, Martin; Phone: +421 904766408;

e-mail: mazuchova.jana@gmail.com 
(14). Proper functioning of the peripheral venous system depends on vessels patency, proper functioning of vein valves and muscle pumps. The vein valves are essential in assuring that blood flows in the correct direction, especially in the standing position. There is a series of one-way bicuspid valves located throughout the deep and superficial venous system. Their opening allows the blood flow toward the heart and closing prevents its return toward the feet. $(4,15)$. The failure of valves located at the junctions of the deep and superficial systems (saphenofemoral and saphenopopliteal junctions) allows high pressure to enter the superficial veins. In this situation, venous dilatation and varicose veins start to form and propagate from the proximal junction site down the extremity. High pressure can also enter the superficial system as the consequence of the perforator valves malfunction (4) In the normal state most perforators are inactive. In the presence of venous hypertension, they open up and reflux the blood from deep to superficial veins (14)

Very interesting is the Gräub et al. study (2014) which is focussed on the number of venous valves and the degree of their deterioration in great saphenous veins of patients with CVD in term of their clinical classification. The authors noticed a negative correlation between age and the number of valves. They detected a possitive correlation between $\mathrm{C}$-class (CEAP classification) and increasing age, but no significant correlation between the average number of valves per meter and C-class. Also, valve disease class was positively correlated with the C-class. Therefore further quantitative and qualitative investigation of venous valves in patients with CVD is needed (16).

Structural failures such as valve weakness and wall dilatation in saphenous veins result in retrograde venous blood flow in lower extremities called reflux (17). Reflux in asymptomatic and mildly symptomatic patients is usually isolated and segmental. In patients with skin changes and ulceration, reflux is usually multisegmental and often involves deep, perforating, and superficial veins (18). Naoum and Hunter (2007) found no demonstrable truncal saphenous reflux and, therefore, suggest that observed varicosities were the result of isolated perforating and nonsaphenous vein incompetence (19).

Valves dysfunction in the deep venous system is most often a consequence of previous deep vein thrombosis. Obstruction of the deep veins may limit the outflow of blood, causing an increase of venous pressure with muscle contraction, and lead to the secondary dysfunction of muscle pump. Dysfunction of the muscle pump appears to be a significant mechanism for the development of complication such as leg ulcers (4).

Both reflux or obstruction can lead to the increased distal venous pressure. (3, 17). Venous hypertension or a failure to reduce venous pressure leads to distended veins and to the severe forms of CVD e. g. hyperpigmentation, lipodermatosclerosis, and eventual ulceration $(4,8)$. Under the hypertension influence the blood flow slows down in the microvasculature, capillaries dilate and their permeability barrier is damaged. Therefore fluid and plasma proteins easily infiltrate the interstitial space and predispose to the formation of edema (20). Hemosiderin deposits from degraded hemoglobin from extravasated erythrocytes cause pigmentary changes. (21) Proteins, especially fibrin, located around the vessels as cuffs, together with interstitial edema, inhibit oxygen diffusion and metabolic exchange (20). In addition to alterations in the blood vessels and connective tissue, also changes in the lymphatic network and nervous system may occur. Fragmentation and destruction of microlymphatics may further impair extremity drainage whereas dysfunction of local nerve fibers may alter regulatory mechanisms (4). These processes lead to the development of microangiopathy, with serious cosequences (20). Capillary convolution is strongly associated with the most severe stages such as lipodermatosclerosis and healed ulcerations. The pericapillary fibrin cuffs present in the dermis of the gaiter skin has been associated with a risk of developing leg ulcers before clinical signs of lipodermatosclerosis. The halo formation is a consequence of pericapillary edema resulting from capillary leakage (22).

Histologic abnormalities typical for varicose veins include irregular thickening of intima, fibrosis between intima and adventitia, atrophy and disruption of elastic fibers, thickening of individual collagen fibers, and disorganization of the muscular layers that are heteroge- 
neously distributed throughout the great saphenous vein and its tributaries (10). The distensibility of the venous wall is controlled by collagen, elastin, and smooth muscle cells. Tunica media, containing smooth muscle cells, is responsible for wall tone, which is influenced by autonomic nerves and circulating stimulants. Collagen and elastin are providing for passive tone (23). Structural changes of the vein wall contribute to pathological weakening and result in the venous dilatation. Overproduction of collagen type I decreased synthesis of collagen type III, and disruption of the elastin fibers and smooth muscle cells arrangement have been observed in histological studies of varicose veins segments (24). The total elastin content in varicose veins is reduced, but changes in collagen content are not always significant (25). Aunapuu and Arend (2005) reported that the vein wall was changed in $78 \%$ of the cases with varicose veins. The majority of specimens had either damaged or missing endothelium with the risk of thrombus formation. Smooth muscle cells in media and adventitia were destroyed or showed altered morphology. The amount of connective tissue was increased together with the irregular organization of collagen fibers and disruption of the elastic network around smooth muscle cell bundles. Laminin expression was increased in varicose veins wall and the severity of damage is related to increasing age and female gender (26).

It is certain that both, the pathological alterations in the venous wall and the valves are involved in the early events leading to venous disease. Yet whether the venous wall changes precede the valve insufficiency or venous insufficiency is causing venous dilatation is questionable (3).

The core pathological process of the CVD development is inflammation within the venous circulation that is subjected to increased hydrostatic pressure, therefore resulting in increased ambulatory venous pressure (27). Venous hypertension is thought to induce the associated skin changes through a number of inflammatory mechanisms (8).

Endothelial glycocalyx has several physiological roles in vascular biology. The endothelial dysfunction results from the damage of the glycocalyx and is promoted by inflammatory cells and mediators (28). The inflammatory response involves leukocytes (macrophages, monocytes, T lymphocytes), inflammatory modulators and chemokines, cytokine expression, growth factors, metalloproteinase activity and many regulatory pathways that perpetuate inflammation (27). Endothelial cells on the luminal surface of the blood vessels react to both physical stress, and biochemical stimuli (28). The endothelium and glycocalyx can through the specialized receptors sense changes in shear stress and expression of adhesion molecules which allows the activation of leukocytes (3). Activated leukocytes then during the inflammatory process release proteases and proteinases, free radicals, growth factors, chemokines and inflammatory cytokines into the vessel microenvironment $(25,28)$. Their unbalanced expression results in an environment of persistent inflammation and is responsible for the changes of the venous wall and valves, reflux, venous hypertension, and the progression of tissue destruction and skin changes $(3,28)$. Lymphocyte trafficking in inflammatory processes is mediated by endothelial E- and P-selectins by interacting with lymphocyte selectin ligands (29). Selectins are a family of lectin-like adhesion glycoproteins. The firm adhesion and transendothelial migration of leukocytes are mediated by the interaction of integrins (CD11/CD18, VLA-4) on the surface of leukocytes with immunoglobulin-like adhesion molecules on endothelial cells (ICAM-1, VCAM-1). The expression of P-selectin, E-selectin, ICAM-1 and VCAM-1 on the endothelial surface is temporally coordinated to ensure that the processes of leukocyte rolling and migration can occur for several hours after the initiation of the inflammatory response (30). Activated leukocytes attach to the endothelium, undergo diapedesis, and migrate into the venous wall or valves resulting in the venous wall injury and accumulation of inflammatory cells in the interstitial tissues $(3,25)$. Excessive inflammation and raised levels of proinflammatory cytokines are typical for venous ulcers (31). Activation of the endothelium and stimulation of the leukocytes recruitment and the release of a range of growth factors can stimulate proliferation and migration of vascular smooth muscle cells (25). Proliferation and migration of smooth muscle cells lead to medial thickening and to the formation of neointima, resulting in the 
wall thickening and decrease of lumen space $(32,33)$. It is still unknown if C-reactive protein, inflammatory mediator correlated with neointimal hyperplasia, is a marker of increased risk or a causative agent of the condition (34). Remodeling following migration of immune cells is a critical determinant of vessel patency (35).

Many studies are nowadays pointing to matrix metalloproteinases as important mediators of the degenerative process involved with venous insufficiency. This may be preceded by an inflammatory process which further contributes to venular degeneration and endothelial dysfunction seen in the advanced presentation of disease (36). Matrix metalloproteinases (MMPs), are a family of at least 25 zinc-dependent endopeptidases which can degrade a wide range of substrates, including extracellular matrix components like collagens, laminin, fibronectin, vitronectin, aggrecan, enactin, tenascin, elastin, and proteoglycans (36, 37). They are secreted in the inactive pro-MMPs form by fibroblasts, vascular smooth muscle cells, and leukocytes (38). The MMPs production is stimulated by factors such as oxidative stress (reactive oxygen species, NO), growth factors, and inflammation (cytokines) which lead to its up- or down-regulation with the subsequent remodeling of extracellular matrix $(39,40)$. The increased hydrostatic venous pressure in the lower extremity is thought to induce hypoxia-inducible factors and other MMP inducers such as ECM inducer, prostanoids, chymase, and hormones which lead to increased activity of MMP (41).

MMPs may also affect the endothelium and vascular smooth muscle cells, causing vascular smooth muscle cell migration, proliferation, $\mathrm{Ca}^{2+}$ signalling, and changes in the relaxation and contraction mechanisms of the vein (42).

Varicose veins commonly show increased levels of MMP, but may show no change or decreased levels, depending on the region of varicose veins (atrophic regions with little ECM versus hypertrophic regions with abundant ECM) and MMP form (inactive pro-MMP or active MMP) (41).

Lim and Davies in 2009 observed upregulation of MMP-1, MMP-2, MMP-3, MMP-7 and MMP-9, and tissue inhibitor of metalloproteinase TIMP-1 and TIMP-3 in varicose veins (25). Gomez et al. results showed a significant increase in the content of collagen in large diameter varicosities, where MMP-1 and MMP-2, responsible for its degradation, were significantly reduced. Imbalance in vascular vein remodelling by decreasing the ratio MMP/TIMP could result in collagen accumulation in varicose veins. This mechanism could be protective in order to restrain the blood stasis by reinforcing the vein wall, avoiding ectasic segment formation and venous wall rupture (43). According to Görmüs et al. (2014), the upregulation of elastin expression may play an important role in the pathogenesis of primary varicose veins and, the up-regulation of MMP-2 expression was strongly correlated with hyperlipidemia associated with varicose veins (44).

In 2014 Gomez et al. published the study about control of MMPs expression by PGE $_{2}$ during the wall remodelling of varicose veins. Besides remodelling, prostaglandin $\mathrm{PGE}_{2}$ is also involved in the control of vascular tone, inflammation, and pain. Results of the study have shown that $\mathrm{PGE}_{2}$ could be responsible for the wall thickening as the counteract to venous stasis and for collagen overexpression. Reduced content of $\mathrm{PGE}_{2}$ and lower density of its receptor is responsible for MMP/TIMP ratio downregulation in varicose veins. However, the metabolism and effects of bioactive lipids like prostaglandins and thromboxane have been poorly investigated in the context of varicose veins (43).

Oxygenation of the vein wall is achieved by oxygen diffusing from luminal blood and vasa vasorum (45). Hypoxia together with high hydrostatic pressure can induce an increase in the thickness of the tunica media and intima. To fit this remodeling of the vessel wall there is a secondary expansion of vasa vasorum (46). In the varicosities, the whole media is oxygenated by vasa vasorum compared to only the outer two-thirds of media of normal veins. This suggested that the oxygenation of the outer two-thirds was provided by vasa vasorum and inner one-third of the vein wall by luminal blood. The relative oxygenation and nourishment of the vascular wall may vary depending on several factors including the type and size of the vessel and luminal oxygen tension (45). In areas of intimal hyperplasia, vasa 
vasorum were found to penetrate the whole media as compared to only the outer two-thirds of the media in normal segments of veins. The 3D network of vasa vasorum implies that these layers are metabolically highly active and, therefore, need a continuous blood supply (47). Studies using cell cultures and ex vivo explants demonstrated that leukocytes and endothelium activated by hypoxia released mediators regulating vein wall remodeling are similar to those observed in varicosities (45). Among the regulatory factors of vessel tone, synthesizes, pro- and anti-inflammatory, adhesion molecules and the transcription factor hypoxia-inducible factor-1 alpha (HIF-1 $\alpha$ ), which are responsible for activation of leukocytes, are very important (48). Hypoxia-inducible factors (HIF-1 $\alpha$, HIF-2 $\alpha$ ) are transcription factors that regulate the expression of several genes of oxygen homeostasis. Lim et al. in 2012 investigated if hypoxia was associated with varicose veins by assessing the expression of HIF- $1 \alpha$, HIF- $2 \alpha$, HIF target genes, and upstream HIF regulatory enzymes. Authors observed an increased activation of the HIF pathway, with upregulation of the expression of HIF- $1 \alpha$ and HIF- $2 \alpha$ transcription factors, and HIF target genes in varicose veins. The data suggest that the HIF pathway may be associated with several pathophysiologic changes in the wall of varicose veins (49).

Adherence molecules like selectins, endothelin-1 are regulating not only the inflammatory infiltration of the vessel wall but as well an oxidative stress. Increased oxidative stress is partly mediated by impaired NO bioavailability due to endothelial dysfunction and NADPH oxidase overexpression. The ability to produce NO is crucial for endothelial cells in order to adapt to the stress conditions (50). Nitric oxide is potent vasodilator causing the smooth muscle relaxation and that inhibit cell adhesion, migration, and proliferation $(51,52)$. It is synthesized by nitric oxide synthase, which has 3 known isoforms: neuronal (nNOS), inducible (iNOS) and endothelial (eNOS) (51). NO synthesis and bioactivity may be reduced by reactive oxygen species (ROS). ROS are produced in the vessel wall and their production is induced by proinflammatory cytokines (53). Arteries release more nitric oxide than veins do. The effect of nitric oxide is reduced by endothelium-derived elements originating from the cyclo-oxygenase pathway. Vascular smooth muscle cells are more sensitive than arterial smooth muscle cells to both, nitric oxide and endothelin (54). Haviarová et al. (2011) histomorphological and semi-quantitative evaluation of NOS isoforms showed discontinuous and significantly lower expression of all 3 NOS isoforms in tunica media of varicose veins. Lower levels of nitric oxide in varicose vein wall suggest that varicose vasodilatation is due to other until now unknown mechanism (51).

Abnormal events in the cell cycle may also contribute to changes in the structural integrity of the vein wall, thus predisposing to the development of varicosities (55). Variety of apoptotic markers such as Bax, Bcl-2, Bcl-6, Bcl-xl, Bcl-xs, Fas, FasL, NF-kappaB, Ki-67, different caspases, p53 and others have been measured in human varicose veins and the results of these studies are controversial (55-64). In these publications, tested varicose veins originated from different locations and from patients with various clinical stages of chronic venous disease which could explain differences in described results. Regulation of proliferation, migration and apoptosis is multifactorial and involves the complex regulation of proteases, integrins and extracellular molecules (65).

The big rise in the incidence of varicose veins in women occurs during pregnancy and around the time of menopause, when levels of hormones change $(66,67)$. In the time of pregnancy, when estrogen levels are elevated, varicose veins develop and they regress after birth when sex hormones decline (68). Estrogens cause the relaxation and loosening of the bonds between the collagen fibers and an increase in the synthesis of coagulation proteins which may result in the higher risk of venous thrombosis and its consequences. Increased levels of fibrinogen and coagulation factors VII, VIII, IX and X, and von Willebrand factor are typical for the pregnancy (69). Progesterone inhibits smooth muscle cells contraction, which leads to increase of veins capacity, valvular incompetence and vein dilatations disorders. Estrogen has been shown to accelerate the rate of wound healing in elderly patients by attenuating the inflammatory response. The estrogen receptor proteins (ER and ER ) medi- 
ate the actions of estrogen during wound repair by the activation or repression of target gene transcription (31). Since alteration of the estrogen receptor- (ER ) expression could directly enhance the cellular volume of smooth muscle cells and leads to the disorganization of the contractile-elastic units. Therefore, hypertrophy of SMC could induce the weakness of the venous wall (67). Ashworth et al. (2007) investigated in the case-control study whether single nucleotide polymorphisms in the ERbeta gene are associated with venous ulceration in elderly patients (age $>50$ years). Polymorphisms in close proximity to upstream regulatory regions of the ERbeta gene, including the $\mathrm{ON}$ exon and promoter transcribed in inflammatory cells, have shown significant association $(p<0.05)$ with venous ulceration. Therefore common variation in the regulatory regions of the ERbeta gene may predispose to venous ulceration in a Caucasian British population (31).

Despite ongoing progress in understanding the molecular aspects of CVD, therapies for varicose veins are directed toward anatomic and physical interventions. But these approaches do not fully treat the causes of CVD (11). Effective methods of correcting the venous reflux include the surgeries focused at the restoration of the valvular function in the femoral veins. Pokrovski et al. (2014) were able to restore the valvular function in $84 \%$ of surgery cases. In the remote postoperative period they observed valvular competence in $74.6 \%$ of patients. Carrying out such surgeries may significantly improve the course of the disease due to decreasing the malleolar volume, manifestations of symptoms of chronic venous insufficiency, and improving the quality of life (70). A better understanding of the pathophysiology of CVD is an important step in the identification of new potential therapeutic targets and new treatment strategies and personalized therapy (25). The synthetic MMP inhibitors have been developed as a new strategy, now their effects in the treatment of varicose veins need to be examined (41).

\section{REFERENCES}

1. Mazuch J, Čapov I, Kalač J et al. Chirurgické aspekty chronickej venóznej insuficiencie dolných končatín. Martin: Osveta; 2006.

2. Chwała M, Szczeklik W, Szczeklik M et al. Varicose veins of lower extremities, hemodynamics and treatment methods. Adv Clin Exp Med 2015; 24(1): 5-14.

3. Raffetto JD, Mannello F. Pathophysiology of chronic venous disease. Int Angiol. 2014; 33(3): 212 21.

4. Eberhardt RT, Raffetto JD. Chronic venous insufficiency. Circulation 2014;130(4): 333-46.

5. Rabe E, Pannier F. Clinical, aetiological, anatomical and pathological classification (CEAP): gold standard and limits. Phlebology 2012;27 Suppl 1: 114-8.

6. Pannier F, Rabe E. Progression in venous pathology. Phlebology 2015; 30(1 Suppl): 95-7.

7. Coleridge-Smith P, Bergan JJ. Inflammation in Venous Disease. In: Schmid-Schönbein GW, Granger ND. Molecular Basis for Microcirculatory Disorders. Paris: Springer-Verlag; 2003: 489-500.

8. Meissner MH1, Moneta G, Burnand K et al. Meissner The hemodynamics and diagnosis of venous disease. J Vasc Surg 2007; 46 (Suppl S): 4S-24S.

9. Markovic JN, Shortell CK. Genomics of varicose veins and chronic venous insufficiency. Semin Vasc Surg 2013; 26(1): 2-13.

10. Meissner MH, Gloviczki P, Bergan J et al. Primary chronic venous disorders. J Vasc Surg 2007; 46 Suppl S: 54S-67S.

11. Oklu R, Habito R, Mayr M et al. Pathogenesis of varicose veins J Vasc Interv Radiol. 2012; 23(1): 33-9.

12. Segiet OA, Brzozowa-Zasada M, Piecuch A et al. Biomolecular mechanisms in varicose veins development. Ann Vasc Surg 2015; 29(2): 377-84.

13. Navrátilová $Z$. Patients with venous leg ulcus in an outpatient general practicioner. Med. Pro Praxi 2010; 7(6 a 7): 280-283.

14. Subramoniam V, Riju RM, Pradeep J et al. Chronic Venous Disorders of the Lower Limbs: A Surgical Approach. New Delhi: Springer; 2014.

15. Rhoades R, Bell DR. Medical Physiology: Principles for Clinical Medicine. Baltimore: Lippincott Williams \& Wilkins; 2009. 
16. Gräub AB, Naef M, Wagner $\mathrm{HE}$ et al. More great saphenous vein valves - less varicose veins? Vasa 2014; 43(4): 260-5.

17. Surendran S, S Ramegowda K, Suresh A et al. Arterialization and anomalous vein wall remodeling in varicose veins is associated with upregulated FoxC2-D1l4 pathway. Lab Invest 2016 Jan 25. doi: 10.1038/labinvest.2015.167. [Epub ahead of print]

18. Tran NT, Meissner MH. The epidemiology, pathophysiology, and natural history of chronic venous disease. Semin Vasc Surg 2002; 15(1): 5-12.

19. Naoum JJ, Hunter GC. Pathogenesis of Varicose Veins and Implications for Clinical Management. Vascular 2007; 15(5): 242-249.

20. Bolognia JL, Jorizzo JL, Schaffer JFV. Dermatology. Bolognia: Elsevier Health Sciences, 2012, $2776 \mathrm{p}$.

21. Feldman SR, Sangueza OP, Pichardo-Geisinger R et al. Dermatopathology Primer of Inflammatory Diseases. Boca Ranton: CRC Press; 2013.

22. Wollina U, Abdel-Naser MB, Mani R. A review of the microcirculation in skin in patients with chronic venous insufficiency: the problem and the evidence available for therapeutic options. Int $J$ Low Extrem Wounds 2006; 5(3): 169-80.

23. Wali MA, Dewan M, Eid RA. Histopathological changes in the wall of varicose veins. Int Angiol 2003; 22(2): 188-93.

24. Piazza G. Varicose veins. Circulation 2014; 130(7): 582-7.

25. Lim CS, Davies AH. Pathogenesis of primary varicose veins. Br J Surg 2009; 96(11): 1231-42.

26. Aunapuu M, Arend A. Histopathological changes and expression of adhesion molecules and laminin in varicose veins. Vasa 2005; 34(3): 170-5.

27. Chi YW, Raffetto JD. Venous leg ulceration pathophysiology and evidence based treatment. Vasc Med 2015; 20(2): 168-81.

28. Mannello F, Ligi D, Raffetto JD. Glycosaminoglycan sulodexide modulates inflammatory pathways in chronic venous disease. Int Angiol 2014; 33(3): 236-42.

29. Velázquez F, Grodecki-Pena A, Knapp A et al. CD43 Functions as an E-Selectin Ligand for Th17 Cells In Vitro and Is Required for Rolling on the Vascular Endothelium and Th 17 Cell Recruitment during Inflammation In Vivo. J Immunol 2016; 196(3): 1305-16.

30. Granger DN, Senchenkova E. Inflammation and the Microcirculation. San Rafael: Morgan \& Claypool Life Sciences; 2010.

31. Ashworth JJ, Smyth JV, Pendleton N et al. Polymorphisms spanning the ON exon and promoter of the estrogen receptor-beta (ERbeta) gene ESR2 are associated with venous ulceration. Clin Genet 2008; 73(1): 55-61.

32. Vijayan V, Smith FC, Angelini GD et al. External supports and the prevention of neointima formation in vein grafts. Eur J Vasc Endovasc Surg 2002; 24(1): 13-22.

33. Kleinedler JJ, Foley JD, Orchard EA et al. Novel nanocomposite stent coating releasing resveratrol and quercetin reduces neointimal hyperplasia and promotes re-endothelialization. J Control Release 2012; 159(1): 27-33.

34. Danenberg HD, Welt FG, Walker M et al. Systemic inflammation induced by lipopolysaccharide increases neointimal formation after balloon and stent injury in rabbits. Circulation 2002; 105(24): 2917-22.

35. Desai M, Mirzay-Razzaz J, von Delft D et al. Inhibition of neointimal formation and hyperplasia in vein grafts by external stent/sheath. Vasc Med 2010; 15(4): 287-97.

36. Pocock ES, Alsaigh T, Mazor R et al. Cellular and molecular basis of Venous insufficiency. Vasc Cell 2014; 6(1): 24.

37. Sakurai T, Yoshiga D, Ariyoshi W et al. Essential role of mitogen-activated protein kinases in IL17A-induced MMP-3 expression in human synovial sarcoma cells. BMC Res Notes 2016; 9(1): 68.

38. Martano M, Corteggio A, Restucci B et al. Extracellular matrix remodeling in equine sarcoid: an immunohistochemical and molecular study. BMC Vet Res 2016; 12(1): 24.

39. Amin M, Pushpakumar S, Muradashvili N et al. Regulation and involvement of matrix metalloproteinases in vascular diseases. Front Biosci (Landmark Ed) 2016; 21: 89-118.

40. Chen YC, Wu YR, Mesri M et al. Associations of Matrix Metalloproteinase-9 and Tissue Inhibitory Factor-1 Polymorphisms With Parkinson Disease in Taiwan. Medicine (Baltimore) 2016; 95(5): e2672.

41. MacColl E, Khalil RA. Matrix Metalloproteinases as Regulators of Vein Structure and Function: Implications in Chronic Venous Disease. J Pharmacol Exp Ther 2015; 355(3): 410-28.

42. Kucukguven A, Khalil RA. Matrix metalloproteinases as potential targets in the venous dilation associated with varicose veins. Curr Drug Targets 2013; 14(3): 287-324.

43. Gomez I, Benyahia C, Louedec L et al. Decreased PGE content reduces MMP-1 activity and consequently increases collagen density in human varicose vein. PLoS One 2014; 9(2): e88021. 
44. Görmüs U, Timirci-Kahraman O, Ergen A et al. Expression levels of elastin and related genes in human varicose veins. Folia Biol (Praha) 2014; 60(2): 68-73.

45. Lim CS, Gohel MS, Shepherd AC et al. Venous hypoxia: a poorly studied etiological factor of varicose veins. J Vasc Res 2011; 48(3): 185-94.

46. Tao W, Wei H, Rui X et al. High hydrostatic pressure upon the vasa vasorum of the greater saphenous and splenic vein walls: a comparative study. Int Angiol 2015; 34(6): 568-75.

47. Kachlik D, Baca V, Stingl J et al. Architectonic arrangement of the vasa vasorum of the human great saphenous vein. J Vasc Res 2007; 44(2): 157-66.

48. Ghaderian SM, Lindsey NJ, Graham AM et al. Pathogenic mechanisms in varicose vein disease: the role of hypoxia and inflammation. Pathology 2010; 42(5): 446-53.

49. Lim CS, Kiriakidis S, Paleolog EM et al. Increased activation of the hypoxia-inducible factor pathway in varicose veins. J Vasc Surg 2012; 55(5): 1427-39.

50. Siasos G, Mourouzis K, Oikonomou E et al. The Role of Endothelial Dysfunction in Aortic Aneurysms. Curr Pharm Des 2015; 21(28): 4016-34.

51. Haviarová Z, Janegová A, Janega $\mathrm{P}$ et al. Expression of constitutive nitric oxide synthase isoforms in varicose vein wall; preliminary results. Int J Vasc Med 2011; 2011: 204723.

52. Uzarski JS, Scott EW, McFetridge PS. Adaptation of endothelial cells to physiologically-modeled, variable shear stress. PLoS One 2013; 8(2):e57004.

53. Blum A, Ginat-Maimon L, Yehuda $\mathrm{H}$ et al. Inhibition of inflammation may enhance nitric oxide availability in patients undergoing bariatric surgery for weight loss. J Intern Med 2015; 278(4): 401-9.

54. Shami SK, Cheatle TR. Fegan's Compression Sclerotherapy for Varicose Veins. Springer Science \& Business Media; 2003.

55. Kun L, Ying L, Lei W et al. Dysregulated apoptosis of the venous wall in chronic venous disease and portal hypertension. Phlebology 2015 Oct 6. pii: 0268355515610237. [Epub ahead of print]

56. Yongbo X, Wei H, Lei W et al. Changes in levels of apoptosis in the walls of different segments of great saphenous varicose veins. Phlebology 2015 Sep 28. pii: 0268355515605670. [Epub ahead of print]

57. Li H, Han W, Wang L et al. Assessment of apoptotic cells in the wall of thrombophlebitic saphenous vein. Phlebology 2015 Apr 2. pii: 0268355515580474. [Epub ahead of print]

58. Filis K, Kavantzas N, Dalainas I et al. Evaluation of apoptosis in varicose vein disease complicated by superficial vein thrombosis. Vasa 2014; 43(4): 252-9.

59. Simovart HE, Arend A, Lieberg J et al. Associations of NF-kappaB and bax with apoptosis in varicose veins of women of different age groups. Int J Vasc Med 2011; 2011: 639720.

60. Heising S, Giebel J, Ostrowitzki AL et al. Evaluation of apoptotic cells and immunohistochemical detection of FAS, FAS-L, Bcl-2, Bax, p53 and c-Myc in the skin of patients with chronic venous leg ulcers. Int J Mol Med 2008; 22(4): 497-505.

61. Ducasse E, Giannakakis K, Speziale F et al. Association of primary varicose veins with dysregulated vein wall apoptosis. Eur J Vasc Endovasc Surg 2008; 35(2): 224-9.

62. Ducasse E, Giannakakis K, Chevalier J et al. Dysregulated apoptosis in primary varicose veins. Eur J Vasc Endovasc Surg 2005; 29(3): 316-23.

63. Urbanek T, Skop B, Wiaderkiewicz $\mathrm{R}$ et al. Smooth muscle cell apoptosis in primary varicose veins. Eur J Vasc Endovasc Surg 2004; 28(6): 600-11.

64. Galkowska H, Olszewsk WL, Wojewodzka U et al. Expression of apoptosis- and cell cycle-related proteins in epidermis of venous leg and diabetic foot ulcers. Surgery 2003; 134(2): 213-20.

65. Zou Y, Qi Y, Roztocil E, Davies MG. Patterns of gelatinase activation induced by injury in the murine femoral artery. J Surg Res 2009; 154(1): 135-42.

66. Maleskey G, Kittel MS. The hormone connection: revolutionary discoveries linking hormones and women's health problems. New York: Rodale Press; 2001.

67. Somers P, Knaapen M. Angiology. The histopathology of varicose vein disease. 2006; 57(5): 546-55.

68. Mashiah A, Berman V, Thole $\mathrm{HH}$ et al. Estrogen and progesterone receptors in normal and varicose saphenous veins. Cardiovasc Surg 1999; 7(3): 327-31.

69. Ropacka-Lesiak M, Kasperczak J, Breborowicz GH. [Risk factors for the development of venous insufficiency of the lower limbs during pregnancy-part 1]. Ginekol Pol 2012; 83(12): 939-42.

70. Pokrovski AV, Gradusov EG, Ignat'ev IM et al. [Restoration of valvular function of deep veins in lower-limb varicose disease]. Angiol Sosud Khir 2014; 20(2): 80-9.

Received: May,26,2016

Accepted: June,20,2016 